\title{
A Mobile Shopping Assistant to Support Product Domesticity in Consumer Decisions
}

\author{
Sotirios - Foivos Stamopoulos \\ University of Patras \\ Rio, 26500 \\ Greece \\ +306945849202 \\ stamopoulos.fivos@gmail.com
}

\author{
Andreas Komninos \\ University of Strathclyde \\ 26 Richmond St. \\ Glasgow G1 1XH, UK \\ $+441415483911$ \\ andreas.komninos@strath.ac.uk
}

\author{
Ioannis Garofalakis \\ University of Patras \\ Rio, 26500 \\ Greece \\ $+302610997526$ \\ garofala@ceid.upatras.gr
}

\begin{abstract}
In this paper we propose and develop a mobile persuasive application to raise user's interest in specific products, by providing information about each product, focusing on the production country and the origin of the company that produces it. During shopping, the user receives information about each product by optically scanning product barcodes with a mobile device. Recommendations for similar products based on product origin are also provided. In an exploratory evaluation, users indicated that the application was found to be easy to use and was rated useful. Moreover, our experimental results show that the proposed system can significantly affect the decision making process of the users.
\end{abstract}

\section{Categories and Subject Descriptors}

H.1.2 [Models and Principles]: User/Machine Systems - Human Factors, Human Information Processing

H.3.5 [Information Storage and Retrieval]: Online Information Services - Web-based Services

\section{General Terms}

Design, Human Factors.

\section{Keywords}

Mobile Recommendation Systems; Persuasive Technologies; Mobile Commerce

\section{INTRODUCTION}

When confronted with a choice of products in supermarkets, consumers must make decisions on which product to choose. These decisions depend on several factors, of which some are obvious and easily aided by labeling on the packaging and the supermarket aisle. Such factors are prices, quantity, brand type (own-label or independent) etc. However, consumers are additionally influenced by other factors, which depend on their attitude and personal values in wider society. The rise of the "ethical consumer", i.e. a consumer who makes decisions under the awareness of ethical issues has been an ongoing subject of research since the mid-90s [1]. Amongst the factors that influence

\footnotetext{
Permission to make digital or hard copies of part or all of this work for personal or classroom use is granted without fee provided that copies are not made or distributed for profit or commercial advantage and that copies bear this notice and the full citation on the first page. Copyrights for third-party components of this work must be honored. For all other uses, contact the Owner/Author. Copyright is held by the owner/author(s).
}

PCI '14, Oct 02-04 2014, Athens, Greece ACM 978-1-4503-2897-5/14/10.

http://dx.doi.org/10.1145/2645791.2645847 ethical consumption decisions, is the origin of a product (domesticity). The effect of product origin is termed the domestic country bias [2] and it has been shown to be a measurable trait in the creation of personality profiles of consumers (consumer ethnocentricity). It has also been shown that while a domestic country bias does not exist across all product categories, it is strongly important in the food item category. Although research shows domesticity to be important to consumers for the formulation of satisfactory choices, usually this information is not prominently displayed in the packaging and very often this information is hardly legible or difficult to find. The legal status for product origin labeling is still unclear in the EU, with many companies often employing an unclear labeling strategy (for example, "made in EU"), which can lead to confusion as to the true origin of a product. Even when the product is clearly labeled as having been made in a specific country, the decision for consumers is still not as clear-cut. The company who makes the products can be a subsidiary, wholly or partly owned by a "foreign" or multinational company. Hence the true domestic value of a particular product is very difficult for consumers to ascertain.

Lack of information to consumers is a problem that has been extensively treated in literature. The provision of additional information that complements packaging and labeling information has been used in the past to influence consumer behaviour through recommendation of alternative or additional products, although such research is generally based on creating user profiles and recommending items that match these profiles. The profilemaking process and the way recommendations are provided, employ techniques such as collaborative filtering [3] or semantic representation through ontologies [4]. We haven't been able to find in literature so far any applications of mobile technology to help consumers make decisions based on perceived ethical values, apart from [5]. The researchers here provided a mobile app that promotes the purchasing of groceries and vegetables that are inseason and produced locally, through the concept of "ecofriends". The researchers portray the importance of in-season and locally produced goods not as a statement of fact, but as a recommendation that emerges through a user's social network of friends. Through a longitudinal evaluation, it was concluded that the application helped ethically minded consumers make more informed decisions and re-connect the users to food and groceries from their current highly disembodied relation.

In other research, the term "persuasive technology" has been used to describe a class of systems whose target is to change humans' attitudes and behaviors [6]. Such systems have been used with success to motivate users to adopt or further conform to valuebased ethically led lifestyles, by promoting and positively 
rewarding behaviour that is associated with particular values. As an example, such interventions have been successfully evaluated in diverse domains such as the increased use of public transport [7] and the adoption of a healthier lifestyle through exercise [8] and better diets [9].

According to Fogg [10], there is an eight-step process to create successful persuasive technologies, which is:

- Choosing a simple behavior to target - Select the smallest, simplest behavior that matters.

- Choosing a receptive audience - Choose the audience that is most likely to be receptive to the targeted behavior change and they also should be familiar with the technology channel.

- $\quad$ Find what is preventing target behavior - Must pinpoint why people aren't performing the behavior.

- Choose an appropriate technology channel - Which channel is best usually depends on three factors which is the target behavior, the audience, and what is preventing the audience from adopting the behavior.

- $\quad$ Find relevant examples of persuasive technology - Search for examples of successful persuasive technologies that are relevant to their intervention.

- Imitate successful examples - Imitate what's working in the successful examples gathered in fifth step.

- $\quad$ Tests and Iterate quickly

- $\quad$ Expand on success

Furthermore, as referred in [11], there are three principal factors that affect persuasive technology design. These factors are motivation, ability and triggers. In order a target behavior to happen, a person must have sufficient motivation, ability and an appropriate trigger.

With respect to making better decisions while shopping for groceries, we have seen that consumer decisions are motivated by their ethnocentricity but their ability is severely limited by the lack of information about the origin of products and the domestic value of a particular company. Triggers to behaviour modification in our problem domain are provided frequently and namely, at every shopping opportunity. In the following sections we will discuss our approach to empowering users with domesticity information during shopping and the outcomes of our evaluation in a controlled environment.

\section{SYSTEM ARCHITECTURE}

The system we implemented adopts a client-server model and consists of two main parts as shown in Figure 1. The mobile application is installed on a mobile device and can be connected to the server via an HTTP Internet connection. The server hosts the central database where the information that relates companies, products and product barcodes is stored. The central database is implemented in MySQL. For each company and product, we store some basic details and also information on whether the company is domestically owned and each individual product is domestically made. The same server also hosts the Products Data Base Management System (PDBMS), to which only the administrator has access. The PDBMS is implemented as a web application, written in PHP, ensuring that administrators can remotely access and manage the system from any computer.

The mobile application also contains a local copy of the central database, which can be updated on application start, if the user is connected to the Internet. The local database is implemented in
SQLite. For products that are present in the local database, the mobile application is able to report the relevant stored data. The application can continue to work with the locally cached copy of the database, when Internet connectivity is not available. However, when the user starts the application and Internet connectivity is available, the local database can be synchronized with the central database, in order to download all the latest information.

In the case that a user scans a product that is not registered in the database, the application asks them if they want to fill in a form providing the products' information and register it to the mobile devices' database. Then the new record will be uploaded to the central database when the user next synchronizes their local copy to the remote database. The administrator is responsible for inserting, updating and deleting information from the central database. They are also responsible for and checking the validity and correctness of the server's database entries and approving user-submitting information, with an option to modify them before committing them to the central database.

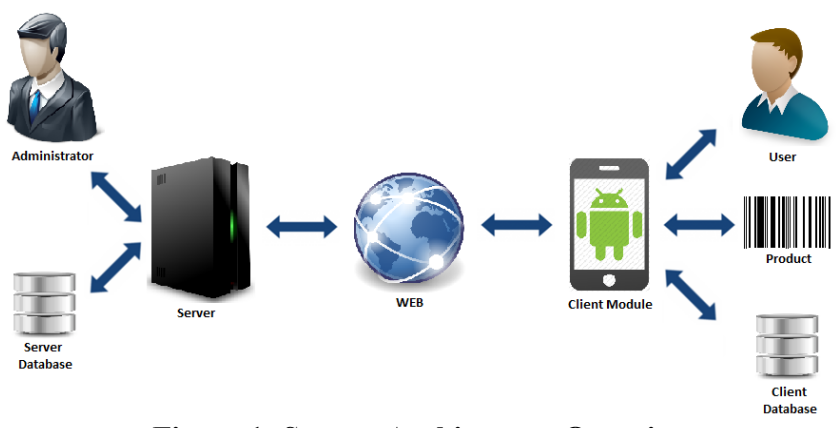

Figure 1: System Architecture Overview

\section{OPERATING PRINCIPLES}

\subsection{Mobile Application Interface}

As shown in Figure 2, the main menu of the application consists of the following four buttons: 'Scan!', 'Companies', 'Products' and 'Update'.

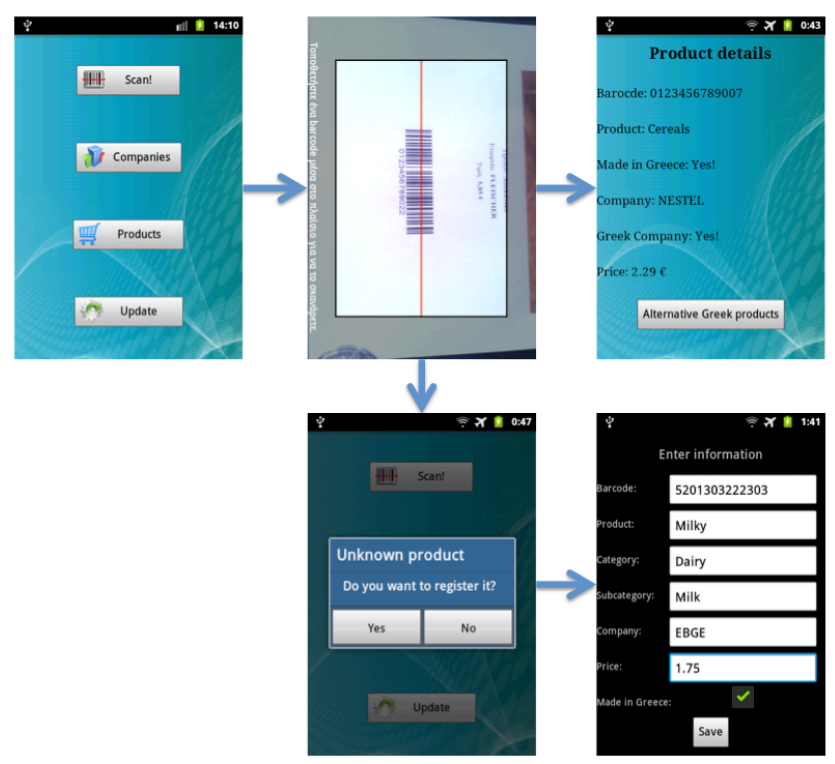

Figure 2: The product scanning process 
By selecting the 'Scan!' button, the integrated android application 'Barcode Scanner' is called and the camera of the mobile device is used as a barcode scanner. Thus, when the user aims with the camera, the barcode of a desired product, our application displays information about it. In case the user scans a product that is not registered in the local database, the application notifies them that the scanned product is not registered and asks if they want to register it. If the user wants to register it then they have to fill in a form providing the product information, by inserting the 'Product name' and selecting the 'Category' and 'Subcategory' to which it belongs. The user also selects the manufacturing 'Company name' from the existing list of companies and also inserts their personal opinion of whether the product is domestically produced. Then by pressing 'Save' button, the new record is registered in the mobile devices' database. Furthermore, the user can add a new company that is not registered in the company list. Upon scanning and based on the product type (whether existing or newly inserted), if a product is not domestically produced or the company that produces it is not domestically owned, the application is able to propose to the user any matching alternative domestic products that are already registered in the database.

By selecting the 'Companies' button, the user is transferred to a list of the names of the companies registered in the central database. Next to the name of domestic companies, we have added the picture of the domestic country flag, so it is easier for the user to set them apart through the list. By selecting a company, the user can navigate to the categories for which the company has products, to the relevant product subcategories and finally to the products as well as the product details of each subcategory. There is also a search field, in case the user wants to find a company by name instead of searching for it through the list.

On selecting the 'Products' button, the user is transferred to a list of all the available product categories (Figure 3). By selecting a category, the user can navigate to its subcategories and to the products as well as the product details of each subcategory. There is also a search field, in case the user wants to find a product by name instead of searching it through the list.

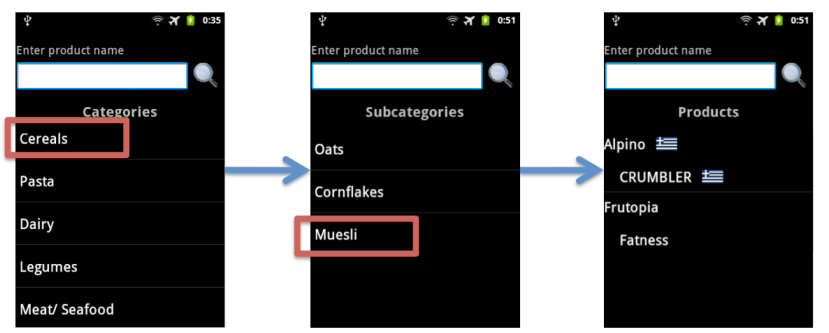

Figure 3: Product browsing process

Finally, by selecting the 'Update' button, the mobile application checks for available networks. In case the mobile device is connected to a network with access to the Internet, the application communicates with the server where the central database is hosted and updates it's local database.

At the same time, if the user has manually entered any new product or company in the mobile application, the record(s) are uploaded to the server where the central database is hosted. Thereafter, the system administrator can check the correctness and validity of the uploaded records and insert them in the primary tables of the central database or delete them. In case that there is no available network, the application informs the user that the update proccess was not completed.

\subsection{Product Database Management System}

The Products Data Base Management System (PDBMS) we implemented consists of a graphical interface through which the administrator can have access to the database. The main page of the interface consists of the following four sections: 'Categories', 'Companies', 'Products' and 'Proposals' (Figure 4). Operation of the PDBMS is not a part of our evaluation hence we will not expand on its use further.

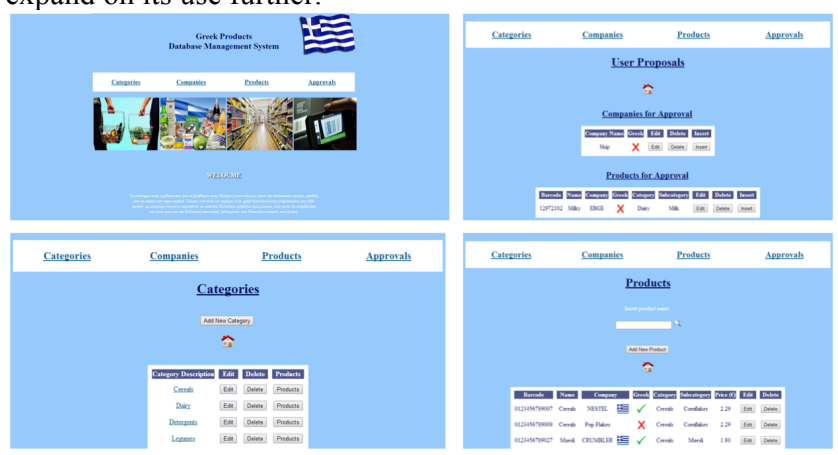

Figure 4: Sample screenshots from the PDBMS

\section{EXPERIMENTAL FRAMEWORK}

\subsection{Experiment Set-up}

To evaluate the effect of our application on shopping behaviour, we designed and carried out a lab-based evaluation using real participants. Within this study, we asked participants to perform a number of shopping tasks with and without the use of the mobile application we developed and compared the results in order to observe whether it can affect the user's decision making process during shopping for groceries.

In total we recruited 32 participants (18 female). The participants' age ranged from 18 to 56 years old. To emulate the shopping environment of a grocery store, we used a room at the University of Patras, organized it in the layout of a small mini market and asked from the participants to "buy" some products according to a shopping list we gave them. Each product of the mini market was represented by an A4 sheet, which contains the picture of the product, its description, the company that manufactures it, its price and barcode. At the bottom of each product sheet, a small container was provided, in which cards containing the product barcode were placed (Figure 5). To "buy" an item, the user simply had to pick one of the barcode cards and place it in a small basket that we provided for them to carry around. Participants were given a generic shopping list of 9 items and were asked to select products for each item on the list. We did not place a time limit, budget or other restrictions on the participants for the shopping tasks. After each purchasing decision and for each product, we asked the user to tell us the main criterion on which their decision was based, choosing one from their estimated Product Domesticity, estimated Quality, Price and estimated Company Domesticity. Finally, because all of our participants were Greek, we chose to represent Greece as the domestic country for consumers in our experiment.

\subsection{Experiment Parameters}

Each participant undertook a shopping task under two conditions, without the help of our mobile app (Phase 1) and with the help of our mobile app (Phase 2). The experiment was not counterbalanced and all participants started with Phase 1, then proceeded to complete Phase 2 . In this case counterbalancing was not required because we wanted to compare self-reported criterion use with actual behaviour first, before proceeding with the 
intervention. To eliminate possible learning effects, shopping lists and products were different for each phase, to eliminate participant bias from their experience in the starting phase.
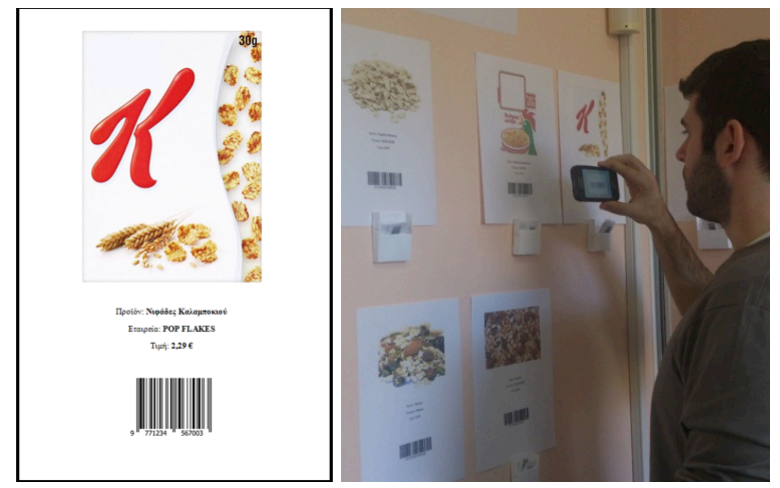

Figure 5: A sample product information sheet and oue study environment

In Phase 1 of the experiment, the participants were asked to "buy" some products according to the shopping list we gave them without any further guidance, as they would do in their daily lives. We had deliberately excluded information on the product sheets indicating whether they are produced domestically or whether the company that produces them is Greek, in order to simulate familiar conditions where such information is often not available or hard to find. Hence for Phase 1, the selection criteria available to each participant were:

- Price

- Product quality (as estimated from the packaging)

- Any assumption that the participant may have made for the origin of the product, based on the product or company name.

In Phase 2, the participants were asked to "buy" some products according to the shopping list we gave them, making use of the mobile application we implemented. The application was installed on a mobile Android device, which was provided to the users as part of the experiment, thus all participants used the same device. The participants were asked to use our application and scan all of the products that suited the shopping list. Thereby the selection criteria for each product were:

- Price

- Product quality (as estimated from the packaging)

- Country in which the product is produced

- Company's origin

For each use case study we used 18 products, 3 product categories and 3 product subcategories. Each category consists of 6 products, which per pair belong to the same subcategory. More specifically, the pairs that belong to the same subcategory are similar products of different company, origin and price but of the same quantity.

As mentioned above, the criteria that participants used in Phase 1 involved a possible assumption of product domesticity, based on the product or company name. For this reason we set the following two parameters in our study:

Concerning the company name criterion, we devised imaginary company names for each product, so as not to affect the participants from potential product choices they makes in their everyday life. Furthermore we separated the companies naming regardless of whether they were Greek or not, in three categories:
- Greek: names that were obviously Greek (e.g. Delphi)

- Ambiguous: acronyms or names that did not hint towards company origin (e.g. $A O M$ )

- Foreign: names that were obviously not Greek (McJoe)

The purpose of this separation is to prevent the user from recognizing if a product is Greek or not, in case he is not using our mobile application.

The second parameter that we used concerns the products pricing. More specifically, we set the following relations between Greek and foreign product prices:

- Greek price $>$ Foreign Price

- Greek price $=$ Foreign Price

- Greek price $<$ Foreign Price

The price deviation between the above relations is $15 \%$. The goal of this parameter is to investigate whether and how the criterion of price plays a role in selecting a product in relation to its origin. For our experiment, we designed the shopping list so that participants were forced to make appropriate decisions involving combinations of all these factors.

Finally, the participants were asked to fill in two questionnaires, one at the start of the experiment and one at the end. The first questionnaire contains general type questions such as gender, participants' age, which mobile device they own, if they download applications and some questions concerning the factors that affect them during the process of product selection. Through these questions we form a shopping behavior profile for each user. The answers of the participants in the latter questions were used as a comparison baseline for their behavior during the experiment. The second questionnaire contained questions related to their experience of using the application during Phase 2. Finally, we should note that during the execution of both experiments we recorded the time that participants needed in order to select each product.

\section{EXPERIMENTAL RESULTS}

\subsection{Initial Attitudes towards Product Choices}

As stated above, we asked the participants about the criteria which usually use while shopping. Their self-reported behavior is depicted in Figure 6 below. It seems that participants are normally mainly interested in quality as estimated by product appearance and price, setting the production country in the third position. These preferences are followed by the company name and finally the origin of the company.

\subsection{Experimental Results}

We then proceeded to examine the experiment parameters, namely the time taken to make a decision, the number of domestic and foreign products chosen and the criteria they used to select products, with an aim to observe any departures from the selfreported behaviour. At first we examined the parameter pair comparisons for normality, using the Shapiro-Wilk test. As observed there is no pair in which both distributions are normal, thus we used only non-parametric tests for the parameters' mean comparisons. After examining the parameter means, we checked whether the difference of the means is statistically significant using a Wilcoxon signed rank test. 


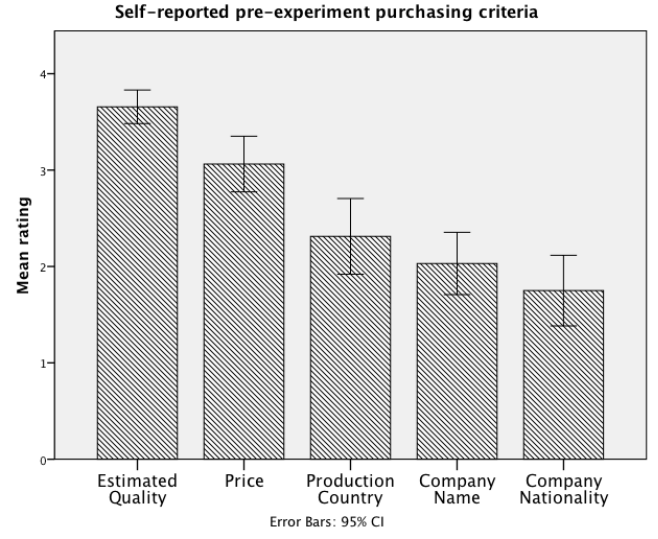

Figure 6: Self-reported pre-experiment purchasing criteria

As shown in Figure 7, the participants needed almost three times more time when used the application $(\mathrm{m}=29.91 \mathrm{sec}, \mathrm{sd}=6.78)$ than without using it $(\mathrm{m}=7.62 \mathrm{sec}, \mathrm{sd}=3.21)$.

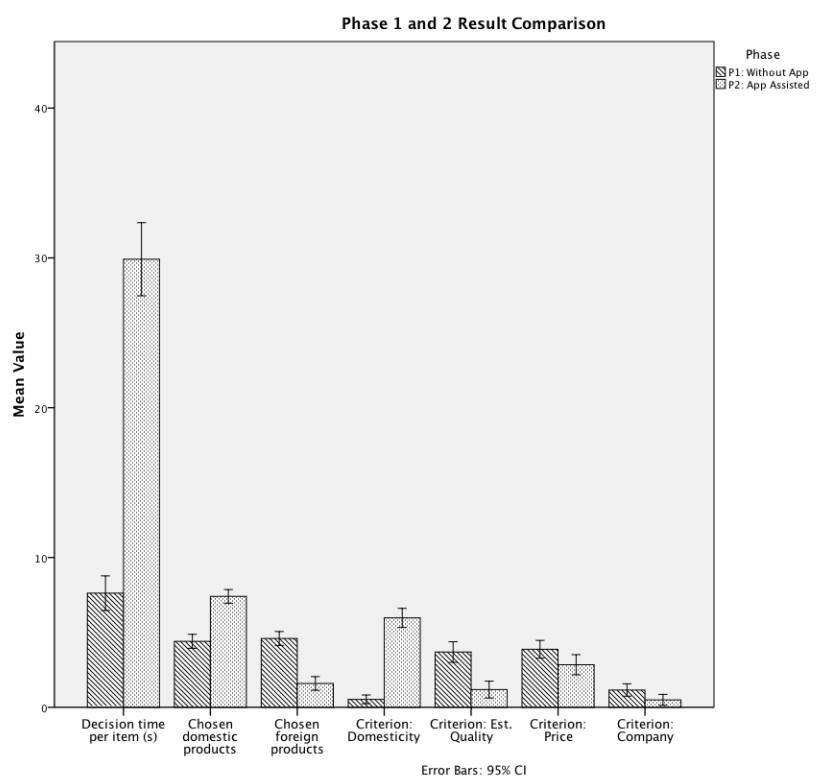

Figure 7: Comparison of Phase 1 and Phase 2 findings

On the other hand, it is observed that the behavior and choices of the participants is affected by the use of the application. In particular, during Phase 2, users selected in most cases domestic products instead of foreign. On average, they selected 4.41 Greek products $(\mathrm{sd}=1.29)$ and 4.59 foreign $(\mathrm{sd}=1.29)$ for the 9 items of the shopping list when the application was not used. However, with the use of the application the products selected were on average 7.41 Greek $(\mathrm{sd}=1.26)$ and 1.59 foreign $(\mathrm{sd}=1.27)$.

As the criterion of the product origin seems to play an important role in the users' behavior change, we investigated for a possible correlation between the uses of this criterion to the final selection of the products, according to their origin. The results of a Spearman correlation analysis show that during Phase 1, the use of the domesticity criterion is not associated with the number of Greek products that were selected ( $\mathrm{p}>0.05)$. In contrast, in Phase 2 , the use of the origin criterion shows a strong and statistically significant correlation to the number of Greek products that were selected $(\mathrm{R}=0.638, \mathrm{p}<0.01)$. This indicates that the preference of participants is strong towards domestic products and the application helps them to choose as such, something that is also confirmed by the subjective evaluation of the usefulness of the application which will be described next.

These observations are in partial contrast with the normal criteria that participants reported at the start of the experiment, where price was reported to be the main factor, and estimated quality (product appearance) second. This is perhaps attributable to the design of our experiment, in which the participant had to judge quality only based on the image of the product, while it is known that the quality of the packaging material and overall physical product characteristics play an important role in assessing the quality of the product itself [12,13]. In Phase 1 , the product domesticity criterion was reported third, which is explained by the relative difficulty of obtaining information for this criterion, in contrast to the price (which clearly displayed on products) and quality (which is appreciable by the appearance of the product). On the other hand we see that when using the application, the selfreported criteria that are mentioned as important in their everyday lives are completely subverted, highlighting the origin of the product as the most important (which was third in the usual preferences), with price, quality and company name following at much lower levels.

\subsection{Subjective Evaluation of the Mobile App}

At the end of the experiment, we asked participatns to fill in a subjective evaluation questionnaire to rate their experience using the mobile app. The answers were on a 5-point Likert scale (1=Completely Agree, 5=Completely Disagree). We asked participants for their opinion on the following statements:

Q1. It was easy to learn how to use the mobile app

Q2. The information displayed was adequate in quantity

Q3. Response time for the display of information was satisfactory

Q4. The interface was clear and easily comprehensible

Q5. The mobile app helped me make better choices

Q6. I would frequently use this app if it was installed on my device

\section{Q7. I would recommend this app to friends or family}

The results are shown in Figure 8. According to participant responses, the app was easy to learn to use. Participants felt that more information could have been displayed on the screen, adding more information that relate to the product, such as price in the current supermarket, nutritional values etc. The response time was deemed satisfactory, as was the clarity and comprehensibility of the interface. Participants felt the app really did help them during the decision process and that they made choices that fit their attitudes towards consumption better. Ultimately, participants felt positively towards the application and felt they would use it frequently, as well as recommending it to friends and family.

\section{CONCLUSIONS}

As stated earlier, we hypothesized that providing the user with specific information on product and company domesticity, we can affect their decisions. The quantitative and qualitative analysis of the data collected from participants in this study suggests that the mobile application we developed affected the users' behavior and choices during shopping. Although the paper describes a relatively small-scale study in a controlled environment, it provides encouraging results that may warrant bigger scale studies. In particular, comparing the results of the two experiments, we observe a sharp increase in the frequency of 
picking domestic products during Phase 2 of our experiment, where the mobile application was used. Even the price factor, which was the primary criterion in both self-reported preexperiment questionnaires and during Phase 1, where the mobile application was not used, seems to play a secondary role in the user options placing as the main purchase criterion the fact whether the product is domestic.

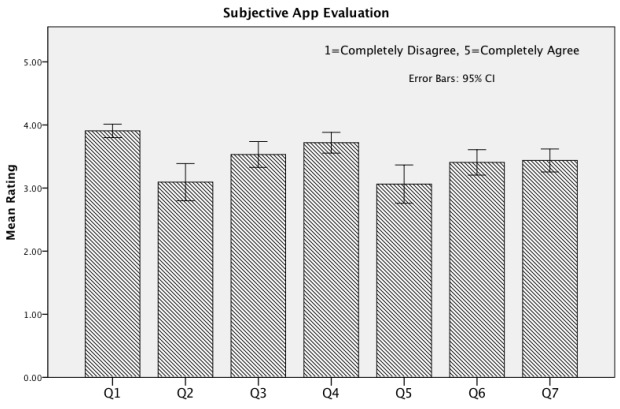

Figure 8: Participants' Subjective Evaluation of our App

These advantages seem to come at a price. We timed the users during shopping in both experiment Phases and we observed that in Phase 2, the users needed on average about 3 times more time in order to complete their shopping. However, feedback from our participants was very encouraging and shows that our idea is useful, simple and perceived as having helped them make better choices.

Our study is limited by the fact that the experiments were carried out in a simulated mini-market environment and not in real conditions. A further limitation was the number of products that were used in the experiment. We could have given participants a bigger shopping list and we would come into a more complete view for the criteria they use when shopping. However, this was not desirable due to the already long duration of our experiment Phases. Moreover, it would be desirable to perform the experiments for a longer period of time, in order to have even more participants and therefore our results to be statistically more accurate. In a longitudinal study, we would expect that shoppers might gradually "learn" about the domesticity of products they are interested in and hence gradually reduce use of our application. A longitudinal study would show if this assumption is true and also would allow us to test alternative ways to keep consumers engaged with the application, such as new recommendations for products, special offers and promotions and the acquisition and exploitation of user profiles, based on scanning habits.

Based on the results of experiments we conducted, we conclude that our mobile application has the potential to significantly affect the decision making process of the users, albeit temporarily for the duration of our experiment. A longitudinal study would also permit us to discover whether such behaviour changes are longlasting.

\section{ACKNOWLEDGMENTS}

We would like to thank Dr. Margarita Komninou for her advice on ethical consumerism and our participants for taking part in our evaluation.

\section{REFERENCES}

[1] Cowe, R. and Williams, S. 2000. Who are the ethical consumers. Co-operative Bank, Manchester, UK.

[2] Balabanis, G. and Diamantopoulos, A. 2004. Domestic country bias, country-of-origin effects, and consumer ethnocentrism: a multidimensional unfolding approach. Journal of the Academy of Marketing Science, 32(1), 80-95.

[3] Nurmi, P., Salovaara, A., Forsblom, A., Bohnert, F. and Floréen, P. 2014. PromotionRank: Ranking and Recommending Grocery Product Promotions Using Personal Shopping Lists. ACM Transactions on Interactive Intelligent Systems (TiiS), 4(1), 1.

[4] Hussein, T., Linder, T. and Ziegler, J. 2013. A ContextAware Shopping Portal Based on Semantic Models. In Semantic Models for Adaptive Interactive Systems (pp. 151-167). Springer London.

[5] Tholander, J., Ståhl, A., Jacobsson, M., Schultz, L., Borgström, S., Normark, M., \& Kosmack-Vaara, E. 2012. But i don't trust my friends: ecofriends--an application for reflective grocery shopping. In Proceedings of the 14th international conference on Human-computer interaction with mobile devices and services (pp. 143-146). ACM.

[6] Wijnand IJsselsteijn, Yvonne de Kort, Cees Midden, Berry Eggen, and Elise van den Hoven. 2006. Persuasive technology for human well-being: setting the scene. In Proceedings of the First international conference on Persuasive technology for human well-

being (PERSUASIVE'06), Wijnand IJsselsteijn, Yvonne de Kort, Cees Midden, Berry Eggen, and Elise van den Hoven (Eds.). Springer-Verlag, Berlin, Heidelberg, 1-5.

[7] Froehlich, J., Dillahunt, T., Klasnja, P., Mankoff, J., Consolvo, S., Harrison, B., \& Landay, J. A. 2009. UbiGreen: investigating a mobile tool for tracking and supporting green transportation habits. In Proceedings of the SIGCHI Conference on Human Factors in Computing Systems (pp. 1043-1052). ACM.

[8] Toscos, T., Faber, A., An, S., \& Gandhi, M. P. 2006. Chick clique: persuasive technology to motivate teenage girls to exercise. In CHI'06 extended abstracts on Human factors in computing systems (pp. 1873-1878). ACM.

[9] Purpura, S., Schwanda, V., Williams, K., Stubler, W., \& Sengers, P. 2011. Fit4life: the design of a persuasive technology promoting healthy behavior and ideal weight. In Proceedings of the SIGCHI Conference on Human Factors in Computing Systems (pp. 423-432). ACM.

[10] BJ Fogg. 2009. Creating persuasive technologies: an eightstep design process. In Proceedings of the 4th International Conference on Persuasive Technology (Persuasive '09). ACM, New York, NY, USA, , Article 44, 6 pages.

[11] BJ Fogg. 2009. A behavior model for persuasive design. In Proceedings of the 4th International Conference on Persuasive Technology (Persuasive '09). ACM, New York, NY, USA, , Article 40, 7 pages.

[12] Louw, A. and Kimber, M. 2006. The Power of Packaging, The Customer Equity Company, United Kingdom [retrieved from http://www.scribd.com/doc/91807833/The-Power-ofPackaging].

[13] Gonzalez-Miranda, S., Alcarria, R., Robles, T., Morales, A., Gonzalez, I., and Montcada, E. 2013. Future Supermarket: Overcoming Food Awareness Challenges. In Innovative Mobile and Internet Services in Ubiquitous Computing (IMIS), 2013 Seventh International Conference on (pp. 483488). IEEE 